\title{
SERS Substrates by the Assembly of Silver Nanocubes: High-Throughput and Enhancement Reliability Considerations
}

\author{
Oded Rabin ${ }^{1,2}$ and Seung Yong Lee ${ }^{1}$ \\ ${ }^{1}$ Department of Materials Science and Engineering, University of Maryland, College Park, MD 20742, USA \\ ${ }^{2}$ Institute for Research in Electronics and Applied Physics, University of Maryland, College Park, MD 20742, USA
}

Correspondence should be addressed to Oded Rabin, oded@umd.edu

Received 3 February 2012; Accepted 21 March 2012

Academic Editor: Mustafa Çulha

Copyright (C) 2012 O. Rabin and S. Y. Lee. This is an open access article distributed under the Creative Commons Attribution License, which permits unrestricted use, distribution, and reproduction in any medium, provided the original work is properly cited.

\begin{abstract}
Small clusters of nanoparticles are ideal substrates for SERS measurements, but the SERS signal enhancement by a particular cluster is strongly dependent on its structural characteristics and the measurement conditions. Two methods for high-throughput assembly of silver nanocubes into small clusters at predetermined locations on a substrate are presented. These fabrication techniques make it possible to study both the structure and the plasmonic properties of hundreds of nanoparticle clusters. The variations in SERS enhancement factors from cluster to cluster were analyzed and correlated with cluster size and configuration, and laser frequency and polarization. Using Raman instruments with $633 \mathrm{~nm}$ and $785 \mathrm{~nm}$ lasers and linear clusters of nanocubes, an increase in the reproducibility of the enhancement and an increase in the average enhancement values were achieved by increasing the number of nanocubes in the cluster, up to 4 nanocubes per cluster. By examining the effect of cluster configuration, it is shown that linear clusters with nanocubes attached in a face-to-face configuration are not as effective SERS substrates as linear clusters in which nanocubes are attached along an edge.
\end{abstract}

\section{Introduction}

The assembly of plasmonic nanoparticles is a simple and inexpensive method for the production of nanoscale gaps between metallic surfaces that generate hot-spots (small volumes with intense electric field strength) when illuminated $[1,2]$. The oscillating electric field in the gap can couple to electronic and vibrational modes of molecules present in the hot-spot. A possible outcome of these interactions is a change in the vibrational state of the molecule and the inelastic scattering of a photon-an event that is detected with a Raman spectrometer. Since the inelastic (Raman) scattering rate is approximately proportional to the 4 th power of the amplitude of the electric field at the site of the molecule and the hot-spots typically enhance the field by a factor of $10^{1}-10^{2}$, the Raman spectrum (or Raman map) may be dominated by photons scattered by the few molecules located in the hot-spots, in what is referred to as Surface-Enhanced Raman Scattering or Surface-Enhanced Raman Spectroscopy (SERS) $[3,4]$.

Knowing the geometry of the assembly and the optical properties of the nanoparticle material and the surrounding medium, the enhancement of the electric field on and near the surface of the nanoparticles can be calculated by solving Maxwell's equations [5, 6]. Such calculations, as well as methodic experiments, show that there are many factors that determine whether a nanoscale gap between metallic nanoparticles will act as an effective SERS site. Local geometry parameters, such as the gap size and the curvature of the surfaces, and electronic factors, such as the frequencydependent dielectric constant of the metal, are particularly important [7]. Changes in the structure and composition of the surface away from the hot-spot (at distances comparable to the wavelength) can also affect the enhancement. In addition, the experimentalists' choices regarding wavelength and polarization greatly influence the effectiveness of SERS [8-10]. Lastly, the ability of targeted molecules to reach the hot-spot and their orientation(s) once there also affect the SERS signal. Control over many of these factors requires the engineering of crystals, surfaces, and chemical interactions with nanoscale precision.

The consequence of the strong dependence of the Raman scattering process on experimental conditions and 
almost-atomistic details of the surface is that the SERS enhancement can vary by several orders of magnitude from site to site on a single substrate [11]. For analytical applications, this problem can be surmounted by increasing the area of signal collection to include a large number of hotspots and thus relying on the average enhancement of the signal across the entire sampling area. Reproducible results can be obtained this way using laboratory or commercial SERS substrates [8]. However, a key advantage of SERS over other optical spectroscopy methods is the ability to record molecular spectra at ultra-low concentrations. Analytical applications that require large sampling areas in order to obtain quantitative results do not make good use of the sensitivity range of the SERS process. Towards creating SERS substrates for quantitative high-sensitivity molecular detection, that is, SERS substrates with high and reproducible enhancements, it is necessary to study the siteto-site variability of the SERS signal of nanofabricated and self-assembly SERS substrates and to understand the reasons for this variability.

With the advent of methods for the synthesis of noble metal nanoparticles with highly uniform shape and size, $[12,13]$ and the demonstration of single molecule SERS sensing using nanoparticle clusters as enhancing substrates, $[14,15]$ the two remaining challenges towards quantitative SERS using nanoparticle self-assembly are (i) the robust design of the enhancing element - the nanoparticle clusterand (ii) the design of a suitable high-throughput fabrication method. These two challenges are intertwined. The design of the enhancing element refers to the selection of the size and geometric features of the nanoparticle cluster [16-20]. These include the particle size and shape, the number of nanoparticles in a cluster and their relative positions. These parameters will need to be tailored specifically for the analyte and the Raman spectrometer to be used. The robustness of the design refers to whether a set of analytical measurement targets (e.g., a 5:1 signal-to-noise ratio when probing a $1 \mathrm{nM}$ sample for $0.5 \mathrm{sec}$ ) is achieved when the tolerances associated with the fabrication method are considered. For example, if the selected cluster design is that of pairs of silver nanospheres and the selected fabrication method produces nanospheres of sizes $55 \pm 5 \mathrm{~nm}$ separated by a $3 \pm 1 \mathrm{~nm}$ gap, a robust design should meet the analytical targets with any likely combination of sphere sizes and gaps that may result out of said production.

Our current research efforts address the robust design and high-throughput fabrication of SERS substrates based on self-assembly of nanoparticle. The focus of this study is on the generation of SERS substrates each consisting of one small cluster of silver nanocubes and the quantification of the enhancement of the SERS signal by these individual clusters. Special procedures were developed in order to quantitatively determine the enhancement factor of hundreds of individual clusters in an efficient manner, without the aid of simulations, and without the need to make assumptions regarding the locations and dimensions of the hot-spots. Using this large pool of data, the robustness of the enhancement by different types of clusters is analyzed.
Recommendations regarding silver nanocube cluster design for SERS are provided.

\section{Experimental Methods for High-Throughput Assembly of Nanoparticles for SERS Studies}

Assemblies of nanoparticles have been examined in great detail by various nanocharacterization tools such as AFM, SEM, and TEM [9, 21-24]. The key technical obstacle for SERS characterization of assemblies of nanoparticles is the need to find and register the locations of the clusters on the substrate in order to perform correlated characterization by Raman spectroscopy and additional characterization tools of the same assemblies [25]. Frequently, the assemblies form in random locations on a substrate, for example, by drop-casting and self-assembly [18]. The substrate needs to be imaged to locate clusters that qualify for the investigation (e.g., isolated from neighboring particles) - a time consuming step that may also alter the effectiveness of the enhancement (e.g., due to radiation damage). As a result, most detailed studies on the SERS effect in nanoparticle clusters have been limited to a small sample population. Alternatively, methods for the preassembly of clusters of nanoparticles in solution have been introduced in recent years, [26] but these clusters often include surface coatings, $[22,24]$ and still need to be deposited and located by scanning the substrate. In order to achieve high-throughput characterization, our focus is on methods that dictate $a$ priori the sites on the substrate in which clusters will form. We present two methods based on (i) vertical deposition [27-29] and (ii) electrophoretic deposition [30] in patterned substrates. In both methods the SERS substrate preparation involves the synthesis of plasmonic nanoparticles as a colloidal solution followed by the templated assembly of the nanoparticles into discrete clusters. The patterned substrates and the deposition method guide the formation of clusters in preselected locations, in this work, a square array with a period (site-to-site separation) of $5 \mu \mathrm{m}$. This pattern geometry was chosen for the time-efficient collection of spectra from hundreds of sites using the $\mathrm{x}-\mathrm{y}$ stage automaticmapping function provided with the control software of the Raman spectrometer (LabRAM Jovin-Yvon microRaman system).

2.1. Vertical Deposition in Patterned Substrates. In the vertical deposition method a substrate is held vertically in the colloidal solution and then pulled out in a vertical motion at a constant velocity. Nanoparticles are deposited on the substrate as the air/liquid interface traverses the surface. When the surface of the substrate contains topographic steps, nanoparticle deposition is enhanced at the bottom of these steps [27-29]. In this work, topographic steps were generated by lithographic patterning and etching. Surface wetting and the presence of a meniscus are favorable conditions for the selective deposition of nanoparticles in the patterns.

The substrates used in the vertical deposition were fabricated out of single-side polished (100) Si wafers (Silicon Inc.). E-beam lithography and reactive ion etching with 
a $\mathrm{SF}_{6} / \mathrm{O}_{2}$ gas mixture $(100 \mathrm{~W}$ power, 50 mTorr pressure, $50 \mathrm{sccm}: 10 \mathrm{sccm} \mathrm{SF} 6: \mathrm{O}_{2}$ flow ratio) were used to generate a square array of nanoscale pores, $200 \mathrm{~nm}$ in diameter, $100 \mathrm{~nm}$ in depth, and $5 \mu \mathrm{m}$ apart. The dimensions of the pores were chosen to accommodate small clusters of $85 \mathrm{~nm}$ silver nanocubes. Larger markers were patterned around the array to allow the user to recognize the position of the columns and rows of the array by optical microscopy. The etched silicon wafer was rinsed with acetone, treated with oxygen plasma, and cleaned with aqua regia to remove any residue from the patterning process [31]. An SEM image of the asprepared substrate is shown in Figure 1. The vertical velocity of the substrate during the deposition and the solvent of the colloidal solution were adjusted to achieve the highest efficiency for filling the pores with nanocubes. The velocities used were in the range of 0.16 to $1.25 \mathrm{~mm} / \mathrm{hr}$. The vertical deposition process took between 3 to 10 hours to complete. The colloidal solutions used in the vertical deposition were obtained by resuspending $85 \mathrm{~nm}$ silver nanocubes passivated by polyvinylpyrrolidone (see Section 3.2) in DI water to a concentration of $2 \mathrm{mM}$ (based on $\mathrm{Ag}$ ).

\subsection{Electrophoretic Deposition in Patterned Substrates. In} the electrophoretic deposition method charged particles are pulled by electric fields between two electrodes in a lowcurrent electrolytic cell [30, 32-35]. In our experiments, the electrolyte consisted of $110 \mathrm{~nm}$ silver nanocubes with a polymeric coating (see Section 3.2) suspended in DI water at a concentration of $0.25-2 \mathrm{mM}$ (based on Ag). No supporting electrolyte was added. The silver nanocubes are negatively charged when suspended in DI water due to the polymer surfactant and can be deposited exclusively on the anode by electrophoresis. A $0.5 \mathrm{~cm}^{2}$ single-side polished (100) silicon wafer was used as the anode and a steel mesh as the cathode. In order to guide the deposition of the nanocubes to chosen sites, the anode was covered with a sacrificial insulating film patterned by e-beam lithography (Scheme 1). Typically, we used a $200 \mathrm{~nm}$ thick PMMA film patterned with an array of $150 \mathrm{~nm}$ by $500 \mathrm{~nm}$ rectangular pores. During electrophoresis, nanocubes near the anode concentrated and deposited in the areas where the silicon surface was exposed to the solution. A constant voltage of $15 \mathrm{~V}$ was applied for a short period of time (10-60 seconds, depending on the concentration of the particles in the electrolyte) to limit the generation of large clusters and minimize parasitic electrochemical reactions. Typical current values were 10-40 $\mu \mathrm{A}$. Dissolution of the PMMA layer removed all nanocubes except those clustered in direct contact with the silicon surface (Scheme 1).

2.3. Comparison between Deposition Methods. It may be worthy to compare attributes of the vertical deposition and the electrophoretic deposition methods for the purpose of generating small clusters of nanoparticles in predetermined locations on substrates.

(i) Duration and Cost. The electrophoretic deposition can be completed in a few minutes, while the vertical deposition is a significantly slower process. However, when the time dedicated to patterning the substrate

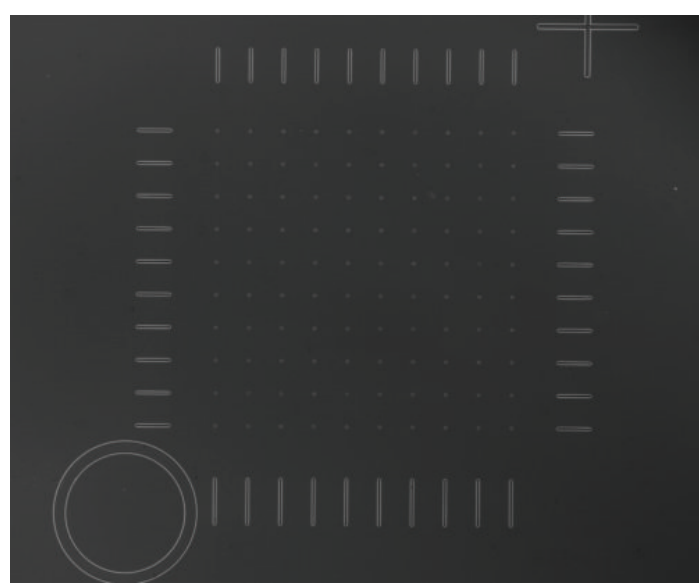

FIGURE 1: SEM image of the patterned silicon substrate used for the vertical deposition process. The pattern includes one hundred circular pores, $200 \mathrm{~nm}$ in diameter, in a square array surrounded by twenty alignment markers identifying the locations of the columns and rows, and auxiliary identification marks (ring and cross). The spacing between adjacent rows and columns is $5 \mu \mathrm{m}$.

is also considered, the vertical deposition saves time and cost with a recyclable substrate that can be reused after cleaning (because the pores are permanently etched into the silicon).

(ii) Filling Efficiency. The electrophoretic deposition is more reliable in generating clusters in the designated sites, while the efficiency of the vertical deposition process depends on the shape of the pore, and on the wettability of the substrate and the volatility of the solvent. The latter are more difficult to test and optimize.

(iii) Damage to Nanocubes. During the electrophoretic deposition, surface reactions involving electroactive species from the solution cannot be excluded, however, to date no detrimental effects on the silver nanocubes were recognized by imaging or spectroscopy. During the vertical deposition, the nanocubes may react on defect sites in the surface of the silicon substrate [31]. These reactions can be prevented by following standard surface cleaning and passivation procedures.

(iv) Aging. The rate of degradation of the nanocubes in ambient air is comparable in both methods. Substrates were usually used within 48 hours of nanocube deposition.

\section{Experimental Methods for High-Throughput SERS Characterization of Assemblies of Nanoparticles}

3.1. The SERS Substrate Enhancement Factor. There are different approaches to calculate the SERS substrate enhancement factor (SSEF), which, regrettably, make it very challenging to compare substrate characteristics and research 


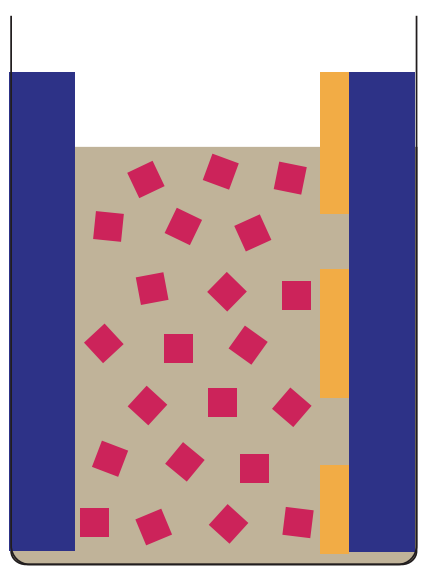

(a)

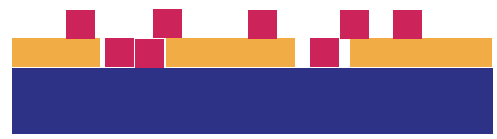

(c)

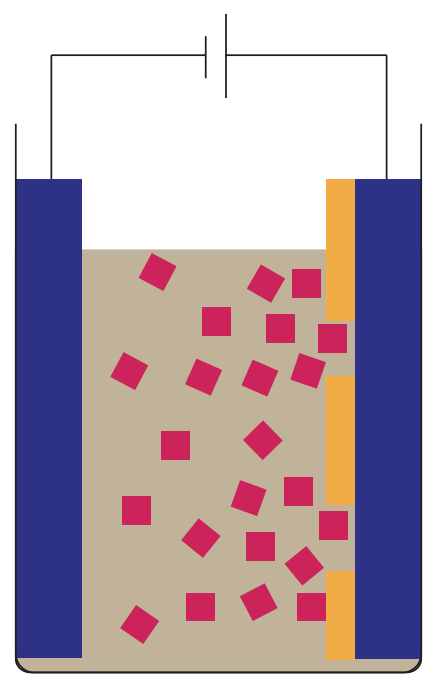

(b)

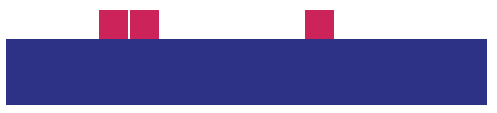

(d)

Scheme 1: Illustration of the electrophoretic deposition process. (a) A patterned electrode and a counter electrode are immersed in a colloidal solution of nanoparticles. (b) An electric field is applied, causing the charged nanoparticles to deposit on one electrode. (c) The deposited nanoparticles are primarily concentrated near the patterned openings in the sacrificial insulating layer. (d) The sacrificial insulating layer is removed, leaving nanoparticles only in the patterns.

data reported by different groups [36]. Considering that the probed molecule may be in many possible locations and orientations during the measurement, the enhancement factor measured experimentally should be considered as an average response. Selective targeting of molecules to the hotspot site may be engineered into the structure and surface chemistry of the SERS substrate, but such examples are rare [37]. Thus, the approach we have taken to generate the most consistent values of SSEF is to saturate the plasmonic surfaces with the analyte and report the average enhancement acting on the ensemble of adsorbed molecules. The number of molecules per cluster in our experiments is on the order of $10^{5}$ to $10^{6}$ when close-packed monolayers are considered. The SSEF values are calculated by comparing the Raman signal from the molecules on the SERS substrate with that from molecules in a concentrated solution $(0.5 \mathrm{M}$ in 1,5 pentanediol, corresponding to $\sim 10^{10}$ molecules), taking into account the number of molecules being probed in each experiment. SSEF values calculated by this approach are reproducible and of informative value to the end-user.

3.2. Silver Nanocubes. Single-crystal silver nanocubes were synthesized by the polyol reduction method in 1,5pentanediol $[23,38]$. The as-prepared nanocubes are terminated by the silver crystal 100 planes and are coated with a passivation layer of polyvinylpyrrolidone (PVP). The nanocube size is tunable between $50 \mathrm{~nm}$ and $150 \mathrm{~nm}$ by the modification of the reaction conditions. We have used suspensions of nanocubes with an average size of $85 \mathrm{~nm}$ and $110 \mathrm{~nm}$ for the reported SERS experiments.
3.3. Cluster Data Collection and Analysis. Each cluster in the array was probed by Raman spectroscopy after the adsorption of a reporter molecule, 4-aminothiophenol, as a saturated monolayer on all accessible silver surfaces (5 molecules per $\mathrm{nm}^{2}$ ). The spectra were collected using a LabRAM Jovin-Yvon microRaman system with a computercontrolled $\mathrm{x}-\mathrm{y}$ scanning stage. Using a $50 \times$ objective (numerical aperture $\mathrm{NA}=0.5$ ) the laser was focused to a spot $2.2 \mu \mathrm{m}$ in diameter. A dispersive grating with 600 lines $/ \mathrm{mm}$ provided a spectral resolution of $\sim 1 \mathrm{~cm}^{-1}$. Measurements with a HeNe laser $(633 \mathrm{~nm})$ took $4 \mathrm{sec}$ at a power of $0.6 \mathrm{~mW}$ and $10 \mathrm{sec}$ at a power of $0.06 \mathrm{~mW}$. Measurements with a diode laser $(785 \mathrm{~nm})$ took $50 \mathrm{sec}$ with a power of $0.4 \mathrm{~mW}$.

The clusters were then imaged by SEM (Hitachi, SU70) to deduce the number of nanocubes in each cluster and the relative arrangement of the nanocubes within the cluster. These will be referred to as "cluster size" and "cluster configuration," respectively, in this paper. Sites with a single silver nanocube were also included in the study. The SSEF is calculated from the scattering intensity (as determined from the Raman spectrum) and the total surface area of the silver deduced from the nanoparticle and cluster size. The SSEF values obtained in each experiment cover a wide range. The sensitivity range of the Raman spectrometer was set to quantify the scattering intensity corresponding to SSEFs in the highest range of $\sim 10^{4}-10^{8}$. Signals weaker than this range were not determined quantitatively and were set to an arbitrary value of 1 , since they can be considered as impractical for analytical measurement purposes. However, no cluster is ignored in the following analysis, regardless of 


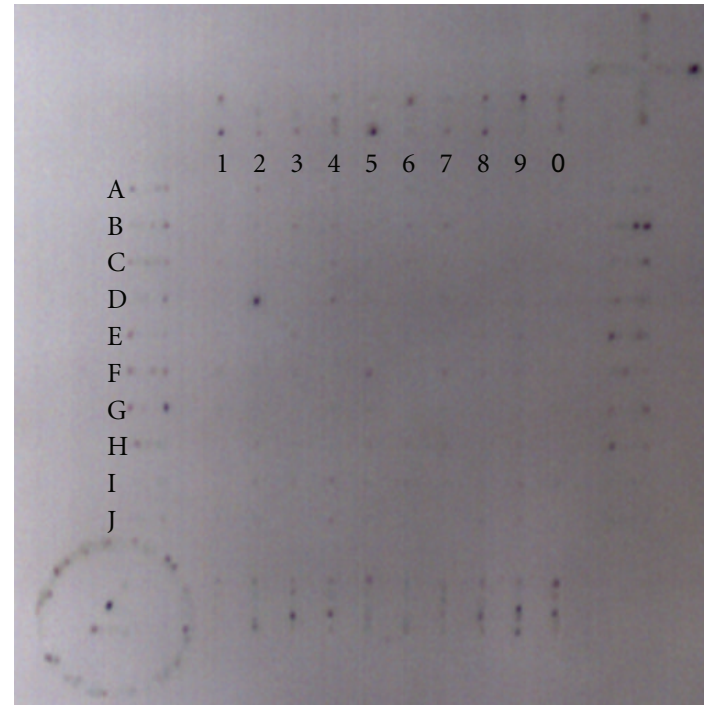

(a)

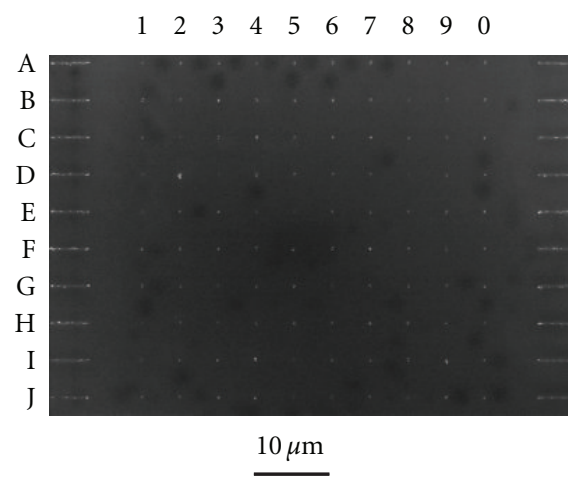

(b)

FIGURE 2: (a) White-light reflectance microscopy image and (b) SEM image of a patterned array of 85 nm nanocube clusters on silicon. The images were scaled to the same magnification. The row (A-J) and column $(1-9,0)$ indicators were added to the images using Adobe Illustrator CS3.

how weak the Raman scattering signal may be. The weak enhancement data are extremely important in analyzing the robustness of the cluster design.

Figure 2 shows an optical micrograph of an array of nanocube clusters side-by-side with the corresponding SEM micrograph of the same sample. The images show the area of the square array in which the clusters are organized, as well as the line markers that indicate the positions of the rows and columns. The line markers, which contain a large number of nanocubes, are clearly visible in the optical microscope image. The light scattering intensity contrast from the array points varies. While some cluster sites are easy to pinpoint (e.g., site D2 is the darkest in the image), others generate no contrast in the optical image (e.g., sites D1 and J0). The SEM micrograph confirms that 89 out of the 100 array sites are occupied with nanocubes; however, the optical image would suggest a lower number of occupied sites. This pair of images illustrates a key point in the study of large number of clusters generated by self-assembly: methods based on the use of light scattering to locate clusters randomly placed on a substrate (e.g. by drop-casting) are biased and may fail to detect a portion of clusters that are weak light scatterers. An analysis based on only a segment of the population of clusters may lead to an unintended bias in the depiction of the dispersion of Raman enhancement values. The formation of clusters in predetermined locations prevents this type of bias in the analysis of self-assembly SERS substrates.

The properties of the clusters were studied for their influence on the robustness of the plasmonic design. Our initial studies have focused on the number of nanocubes in the cluster and the configuration of the cluster. For each analysis, the collection of clusters was divided into $N$ categories based on cluster size or configuration using the SEM data. For each category, consisting of $n_{i}(i=1$ to $N)$ clusters, the SSEF values were collected and sorted from high to low (Figures 3(a) and 3(b)). For the purpose of comparing sets, the sorted SSEF values were plotted against their normalized list index (ranging from $1 / n_{i}$ to 1 ). This normalization is necessary because the number of clusters that fall into each category varies significantly $\left(n_{i} \neq n_{j}\right)$. Figure 3(c) illustrates the trendlines that are obtained by following the above-mentioned data analysis procedure. The $\mathrm{y}$-axis displays the SSEF value and the $\mathrm{x}$-axis can be regarded as the cumulative fraction of the cluster population. A testimony to the robustness of the plasmonic cluster design is for all the clusters in a given category to have similar (and large) SSEF values. Thus, we are seeking plots that show a nearly horizontal trendline.

\section{Results and Discussion}

As part of our investigation of cluster configurations, we have studied the effect of the azimuth orientation of linear silver nanocube clusters on their SERS response. Because of the relevance of this study to the design of the patterned substrates used in all the subsequent experiments, it is presented first.

4.1. The Azimuth Angle of a Pair of Nanocubes. The vertical deposition into circular pores provided us with many isolated pairs of silver nanocubes (dimers) for this study. These dimers are distinguishable due to variations in the gap distance between the cubes (limited by the size of the cavity and the size of the cubes), in the orientation of the dimer axis (i.e., the azimuth angle $\theta$ in the plane of the silicon wafer, measured with respect to the positive $y$-axis, as shown in Figures 4(a) and 4(c)), and in the orientation of the nanocubes in the pair with respect to 


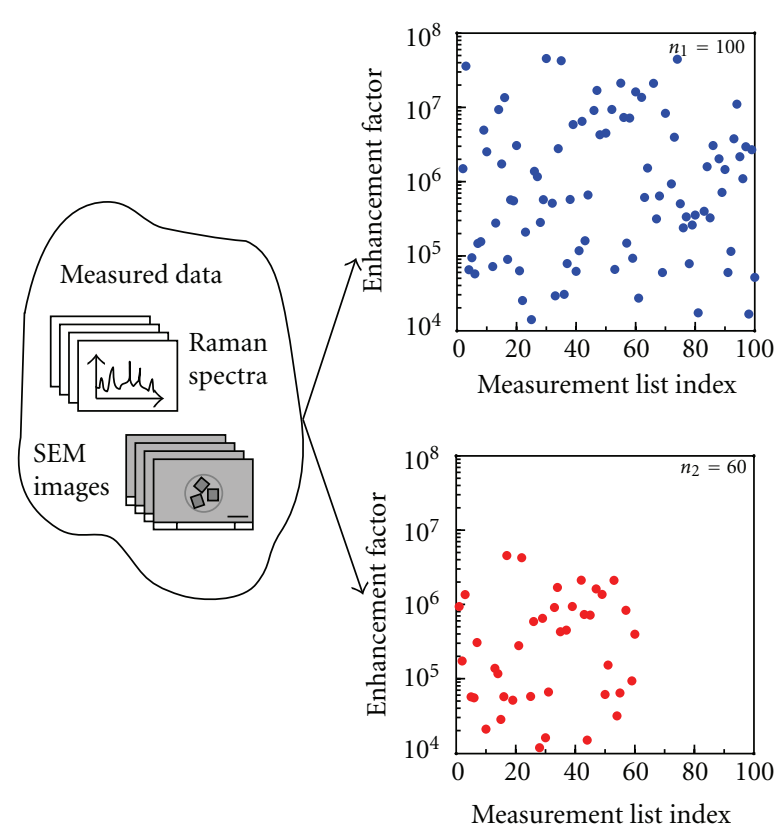

(a) Group data into $\mathrm{N}$ categories (example: $N=2$ )
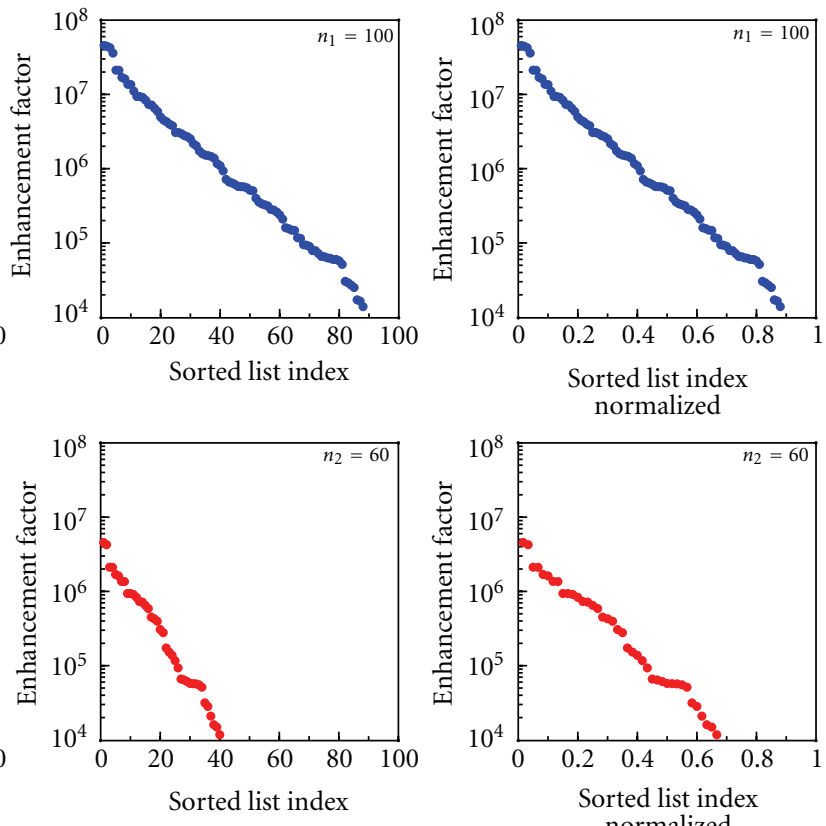

(b) Sort lists

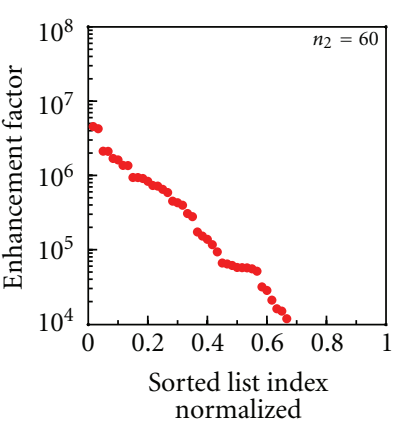

(c) Normalize list index

FIgure 3: Analysis of SSEF data. (a) The data is grouped into $N$ categories $(N=2)$ : set 1 consists of a list of $n_{1}=100$ values (blue), and set 2 consists of a list of $n_{2}=60$ values (red). The list content is plotted against the list index that indicates the sequence in which the data was collected and has no physical meaning. (b) The values within each category are sorted in descending order. (c) The list index is normalized to allow a direct comparison between the lists. The normalized list indices are $0.01,0.02, \ldots, 1$ for set 1 with $n_{1}=100$ (blue) and $1 / 60,1 / 30,1 / 20, \ldots, 1$ for set 2 with $n_{2}=60$ (red). Note that $12 \%$ of the SSEF values in set 1 and $33 \%$ of the values in set 2 are lower than $10^{4}$ and thus are not displayed in any of the plots.

each other. An analysis of the dependence of the SSEF on the azimuth angle of the dimer axis revealed that tight control of this parameter is vital for a robust design. Figure 4 displays the oscillatory trend of the Raman enhancement on the azimuth angle. The root of this behavior is the linear polarization of the incident laser of the Raman spectrometer and the selective coupling between far-field radiation and plasmon modes that are dipolar in nature. The coupling strength is proportional to $|(\vec{D} \cdot \vec{E})|^{2}$, the square of the projection of the $E$-field vector on the direction of the dipole associated with the plasmon mode. In the case of a dimer, the vector $\vec{D}$ is oriented either parallel to the dimer axis (with magnitude $D_{\text {ax }}$ ) or perpendicular to it (with magnitude $\left.D_{\text {orth }}\right)$. When the orientation of the $E$-field vector is set parallel to the $y$ axis of the sample plane, the scattering intensity $I_{y}(\theta)$ is proportional to $\left(D_{\mathrm{ax}} \cos \theta+D_{\text {orth }} \sin \theta\right)^{2}$. When the orientation of the $E$-field vector is set parallel to the $x$ axis of the sample plane, the scattering intensity $I_{x}(\theta)$ is proportional to $\left(D_{\mathrm{ax}} \sin \theta+D_{\text {orth }} \cos \theta\right)^{2}$. If a strong hot-spot exists in the gap between the particles, $D_{\mathrm{ax}} \gg D_{\text {orth }}$ and the $\operatorname{SSEF}(\theta)$ is large and anisotropic. Otherwise, $\operatorname{SSEF}(\theta)$ is weak and more isotropic. These trends are observed in Figure 4(b). The data, plotted as the SERS signal change in response to a $\pi / 2$ change in the incident light polarization versus the dimer axis orientation, are concentrated in two quadrants of the plot. The measurements match well with the theoretical curve that assumes $D_{\text {orth }}=0$. This assumption is justified only if there is good coupling between the plasmons of the nanoparticles in the dimer, and if the excitation wavelength matches the resonance frequency of the coupled plasmon mode. These conditions should also lead to a strong SERS signal. Data from dimers with a strong SERS enhancement therefore fit better to the theoretical curve.

The orientation of the nanocubes of the dimer relative to each other has a more subtle influence on the SSEF [23]. Consequently, our study on the effect of the azimuth angle is fairly insensitive to and unobstructed by variations in the nanocube dimer configuration. In the following studies on the effect of cluster size (Section 4.2) and cluster configuration (Section 4.3) on the SSEF it was important to exclude the effect of variations in the azimuth angle. Thus, in the following experiments only linear silver nanocube clusters of uniform orientation were utilized.

4.2. The Number of Nanocubes in the Cluster. Researchers have undoubtedly demonstrated that strong hot-spots occur at narrow junctions between metallic nanoparticles [2]. There is less certainty regarding the parameters that make those hot-spots most effective towards the SERS process. In particular, a fundamental question which has not yet been 


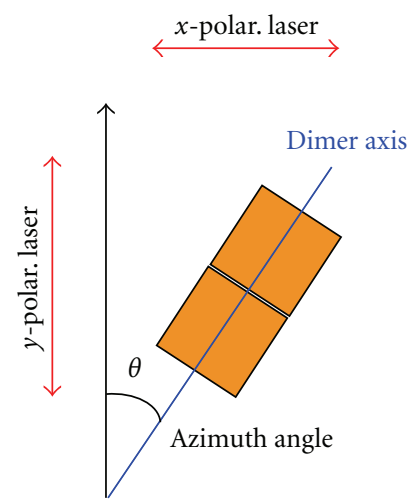

(a)

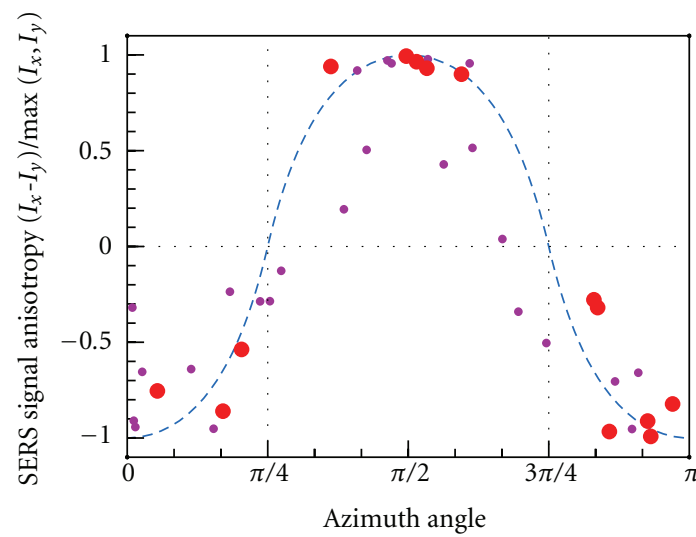

(b)

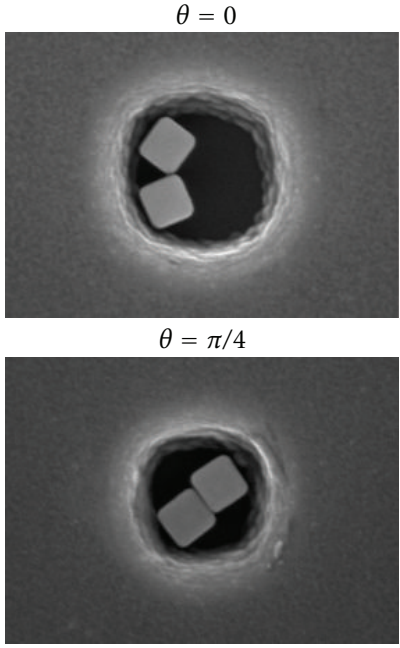

(c)

Figure 4: (a) Schematic of the azimuth angle $\theta$ between the positive $y$-axis and the dimer axis, traced clockwise. (b) The dependence of the SERS signal on the orientation of the polarization of the laser with respect to the orientation of the axis of the dimer of silver nanocubes. The SERS signal anisotropy is calculated as $\left(I_{x}(\theta)-I_{y}(\theta)\right) / \max \left(I_{x}, I_{y}\right)$ (see definitions in text). The theoretical relation $\left(\sin ^{2} \theta-\right.$ $\left.\cos ^{2} \theta\right) / \max \left(\sin ^{2} \theta, \cos ^{2} \theta\right)$ is indicated by the blue dashed line. Data from dimers with SSEF $>10^{5}$ (large circles) is closer to the theoretical trend than data from dimers with SSEF $<10^{5}$ (small circles). (c) Examples of two dimers of silver nanocubes, oriented at $\theta=0$ and $\theta=\pi / 4$, respectively. The nanocube size is $85 \mathrm{~nm}$. Each dimer is located inside a pore.

resolved is whether increasing the number of nanoparticles in a cluster, and therefore the number of junctions between particles, leads to an increase in the enhancement factor. Increasing the number of nanoparticles in a cluster increases the metallic surface area; without a concomitant increase in the scattering intensity the SSEF will decrease. Detrimental retardation effects also become more pronounced as the plasmonic structure increases in size $[39,40]$. Additionally, the change in the cluster size may bring the plasmon mode in or out of resonance with the laser of the Raman spectrometer.

We have studied the effect of the cluster size on the SSEF. To eliminate the effect of the azimuth angle, we have concentrated on the study of linear clusters and therefore have chosen the electrophoretic deposition method to deposit nanocubes within aligned rectangular pores. Representative images of plasmonic clusters consisting of linear assemblies of 2-4 silver nanocubes are shown in Figure 5. The four categories used for the data analysis reflect the number of cubes per site: monomers, dimers, trimers, and tetramers.

While the cluster size has been considered in the context of obtaining the largest enhancement factor, the reproducibility of these SSEF measurements is often overlooked. Indeed, we find that the largest SSEF value recorded for a category of clusters is not a good indicator for the SSEF values of similarly structured clusters. Figures 6(a) and 6(b) present the SSEF data for independently measured clusters of $110 \mathrm{~nm}$ silver nanocubes, using excitation wavelengths of $633 \mathrm{~nm}$ and $785 \mathrm{~nm}$, respectively. The data was grouped by cluster size, with 7-124 clusters per category. The data shows a dramatic difference in the SSEF values of monomers and multiparticle clusters. Monomeric silver nanocube SERS substrates lack the narrow gaps that effectively generate hotspots. The SSEF values of monomers are the lowest, and at least $80 \%$ of the monomers have an SSEF value lower than $10^{4}$. The comparison between enhancements by dimers, trimers, and tetramers leads to the conclusion that the most notable advantage of engineering larger clusters is that the reproducibility of the measurements is improved. The slope of the trendline decreases with cluster size. The trendline for tetramers is the most horizontal. The incorporation of additional nanocubes into the clusters results in improved robustness for the linear-cluster SERS substrate design. Similarly, the portion of unusable clusters diminishes as clusters contain more nanocubes. Of the dimers, at least $10 \%$ at $633 \mathrm{~nm}$ and $60 \%$ at $785 \mathrm{~nm}$ were ineffective SERS substrates with SSEF below $10^{4}$. In comparison, we have not detected tetramers with SSEF below $10^{4}$. The robustness of the trimeric structures is in between that of the tetramers and the dimers.

The data of Figure 6 indicate that the SSEF reproducibility is sensitive to the excitation wavelength. When a plasmon resonance matches with the excitation laser frequency, a boost in the SERS signal is plausible. While the resonance frequency of the plasmon modes depends on the cluster configuration, particularly on the gap sizes over which there is very limited control in the self-assembly methods used here, these frequency variations are small compared to the red-shift occurring when an additional nanoparticle is added to the cluster. Thus, while dipolar plasmons in dimers resonate near the $633 \mathrm{~nm}$ wavelength, they are far from resonance with the $785 \mathrm{~nm}$ wavelength. For this reason the trendline for dimer SSEF values slopes down more 


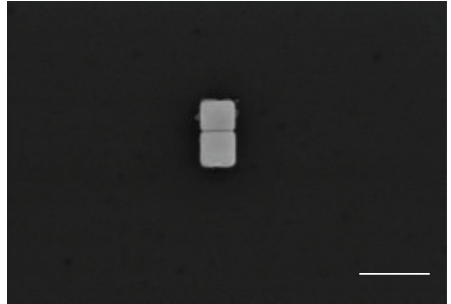

(a)

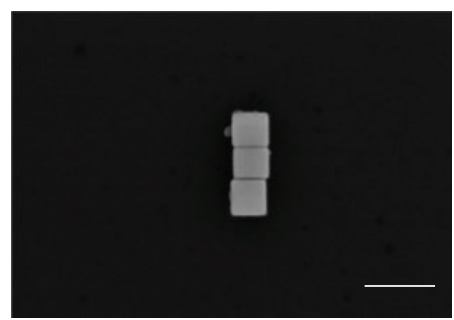

(d)

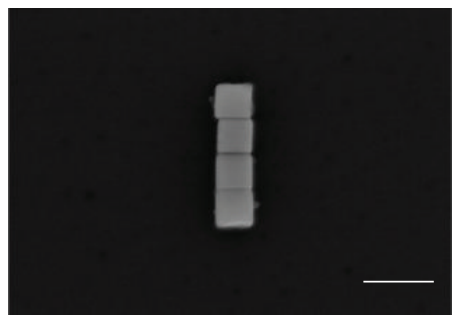

(g)

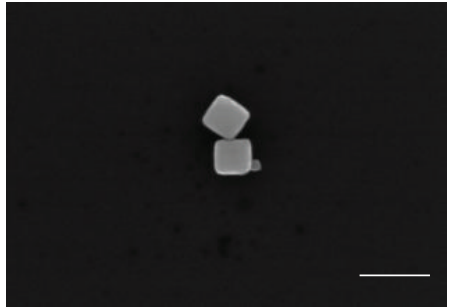

(b)

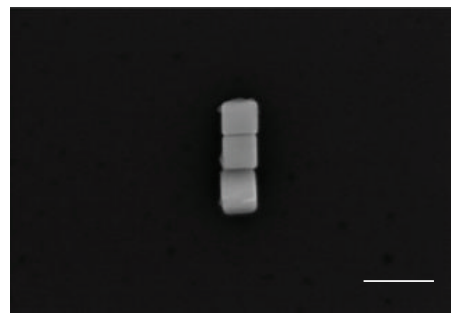

(e)

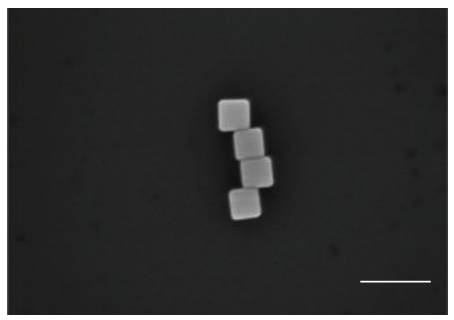

(h)

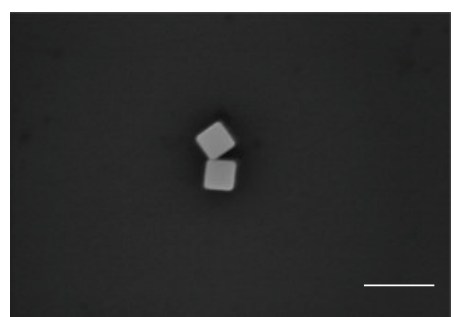

(c)

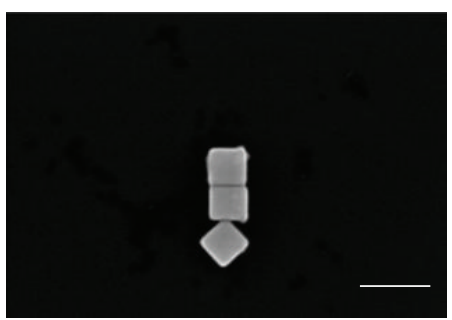

(f)

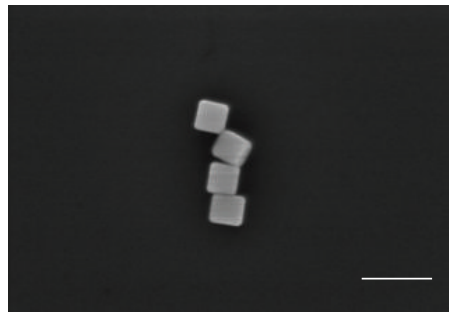

(i)

FIGURE 5: Collection of SEM images of silver nanocube dimers, trimers, and tetramers on silicon formed by the electrophoresis deposition method. The scale bar size is $200 \mathrm{~nm}$. Images (a), (d) and (g) show examples of nanocube clusters with face-to-face configuration.

sharply as we increase the wavelength of the excitation laser (Figure 6(b) versus Figure 6(a)) and the enhancement of a large portion of the dimers decreases below the practical threshold. A similar trend is seen with monomers which resonate near $520 \mathrm{~nm}$.

The number of trimers and tetramers studied is not large enough to reliably determine that the maximum SSEF achieved with the larger clusters is lower than that of dimers. However, the data collected thus far clearly indicates that the enhancements from the larger clusters are more reproducible and less susceptible to the lack of precise positioning control during assembly (i.e., structural variations).

A few monomers have surprisingly high SSEF values $>10^{4}$. We postulate the presence of a hot-spot in the junction between the nanocube and the silicon wafer. Using chemical manipulations designed to direct more molecules to this junction, we were able to increase the percentage of monomers with SSEF $>10^{4}$ to $40 \%$ (see Section 4.3).

\subsection{The Relative Position and Orientation of the Nanocubes} in the Cluster. Several experimental results and theoretical calculations with nanocube dimers have predicted the largest plasmonic enhancements for nanocubes arranged face-toface with a gap of few nanometers between them [23, 41]. Calculations show that strong fields are predicted to extend over the entire face of the nanocube forming the narrow gap. In other configurations the strongest fields are located along cube edges or near its corners. In other words, in the face-toface configuration the hot-spot contains more silver surface area than in other configurations, and thus more signalgenerating molecules experience the strongest fields.

Our results do not reproduce these expectations. We have alluded to this result in our previous study on dimers in circular pores [23], and new data from SERS substrates produced by the electrophoretic method (Figure 5) reinforce the unexpected trend. The analysis of the new data proceeded by dividing the plasmonic structures into 3 categories of clusters: (1) clusters with nanocubes attached face-to-face (FF), (2) clusters of nanocubes attached in a non-face-toface manner (non-FF), that is, along an edge, and (3) clusters of any configuration with at least one gap between the nanocubes that could be resolved by SEM (i.e., gap $>3 \mathrm{~nm}$ ). The gaps between nanocubes in the clusters selected for the first two categories were all less than $3 \mathrm{~nm}$. This categorization was implemented with data sets from nanocube dimers and nanocube trimers, as shown in Figures $7(\mathrm{a})$ and 7(b). The SSEF values of face-to-face (FF) dimers are collectively lower than those of non-face-to-face (non-FF) dimers and the SSEF values of FF trimers are collectively lower than those of non-FF trimers. Strikingly, the FF clusters are comparable 


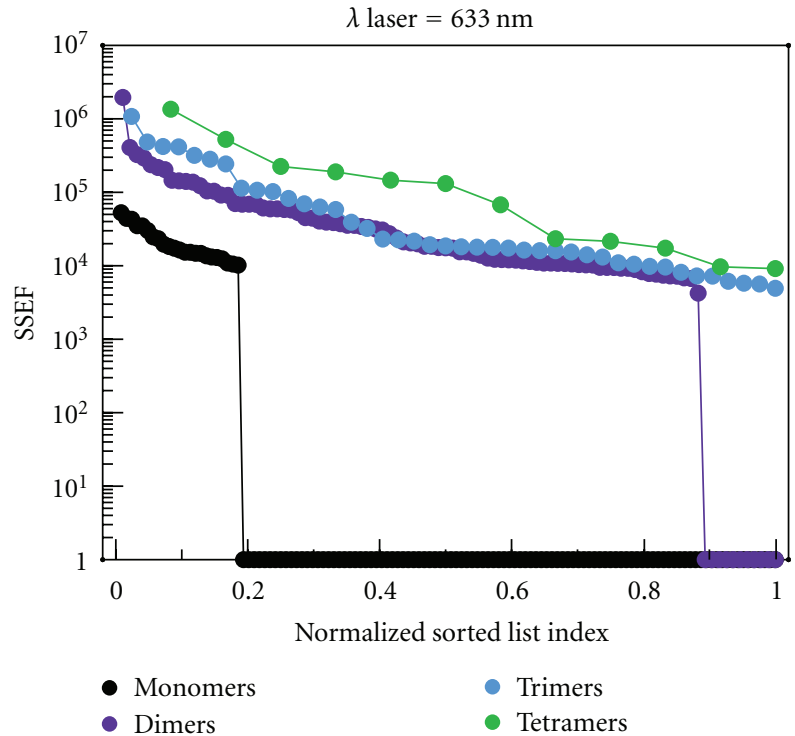

(a)

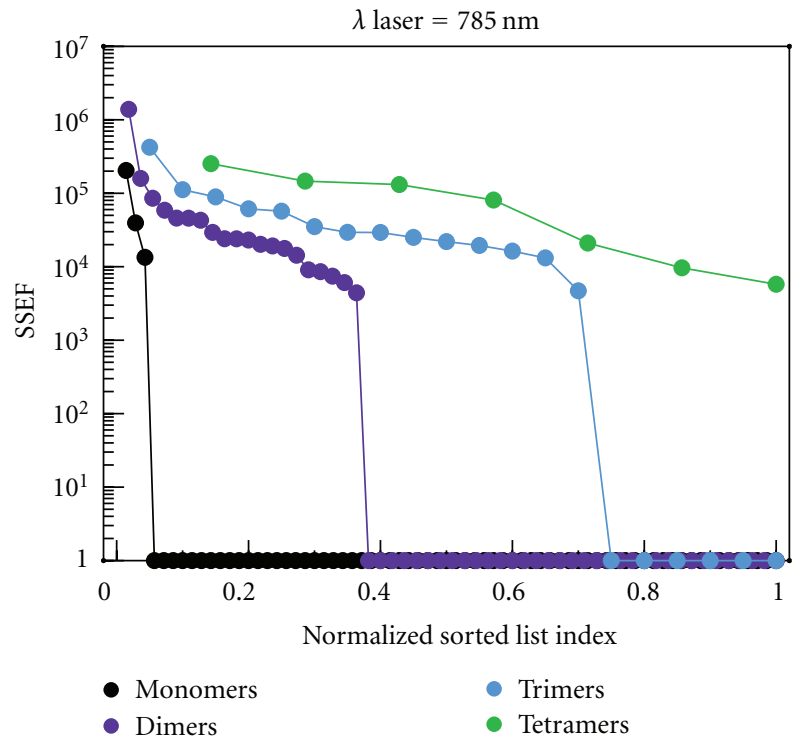

(b)

Figure 6: Cluster size analysis. Sorted SSEF data from silver nanocube monomers (black), dimers (violet), trimers (cyan), and tetramers (green). Excitation laser wavelength: (a) $633 \mathrm{~nm}$ and (b) $785 \mathrm{~nm}$. Nanocube size: $110 \mathrm{~nm}$.

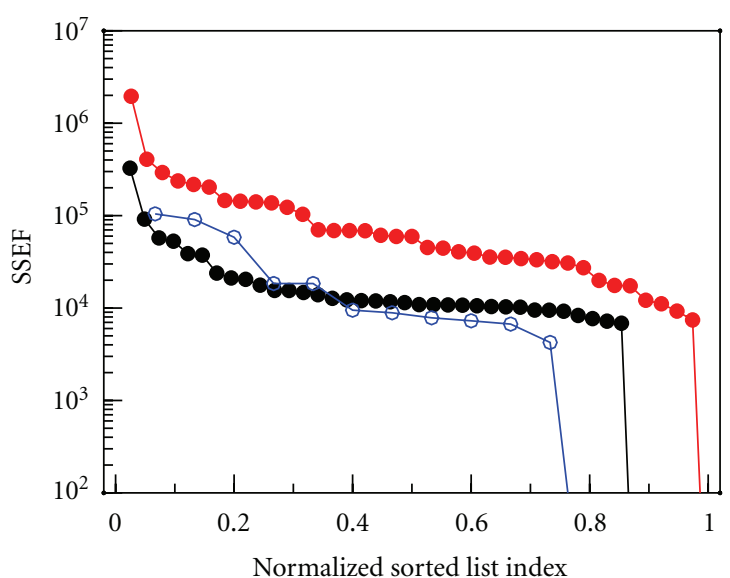

- FF gapless dimers

- Non-FF gapless dimers

o Dimers with a gap

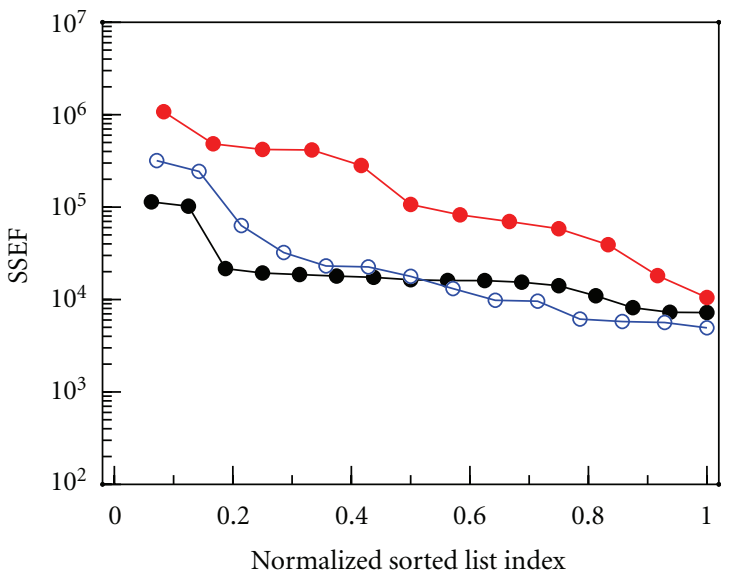

- FF gapless trimers

- Non-FF gapless trimers

- Trimers with a gap

(a)

FIGURE 7: Cluster configuration analysis. Sorted SSEF data from (a) silver nanocube dimers and (b) silver nanocube trimers. The categories are FF clusters with no gaps (black), non-FF clusters with no gaps (red), and clusters with a gap (blue, open circles). Excitation wavelength: $633 \mathrm{~nm}$. Nanocube size: $110 \mathrm{~nm}$.

in their SSEF to clusters in which we can observe a sizeable gap $(>3 \mathrm{~nm})$.

The data in Figure 7 indicate that the FF-type junctions produce smaller Raman scattering enhancements than the non-FF junctions. There are several possible explanations for these observations. The most intuitive hypothesis is that there are no Raman dye molecules in the FF-type junctions. The Raman dye molecules are more likely to adsorb on the metal surface areas that are more accessible to the solution.
The dye solution cannot easily access the surfaces that form the FF-type junctions, since these faces are separated by a gap less than $3 \mathrm{~nm}$ in size that is also occupied by surfacepassivating PVP molecules. To examine the hypothesis that the FF-type clusters have an average low SSEF because of the low surface concentration of the Raman reporter in their gaps, we have compared the SSEF data reported in Figure 6 and Figure 7 with SSEF data obtained from clusters formed with nanocubes that have been treated by adsorption of 


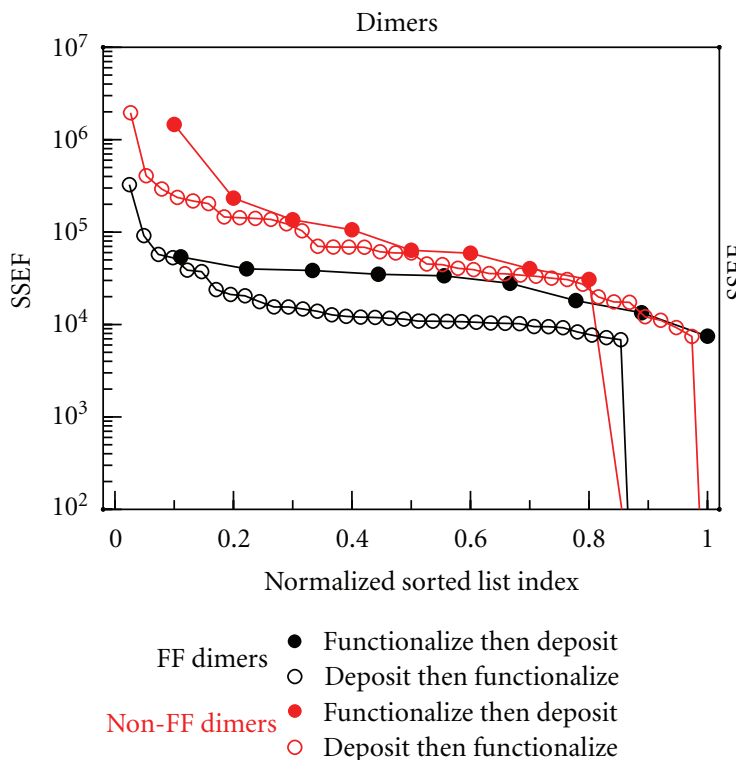

(a)

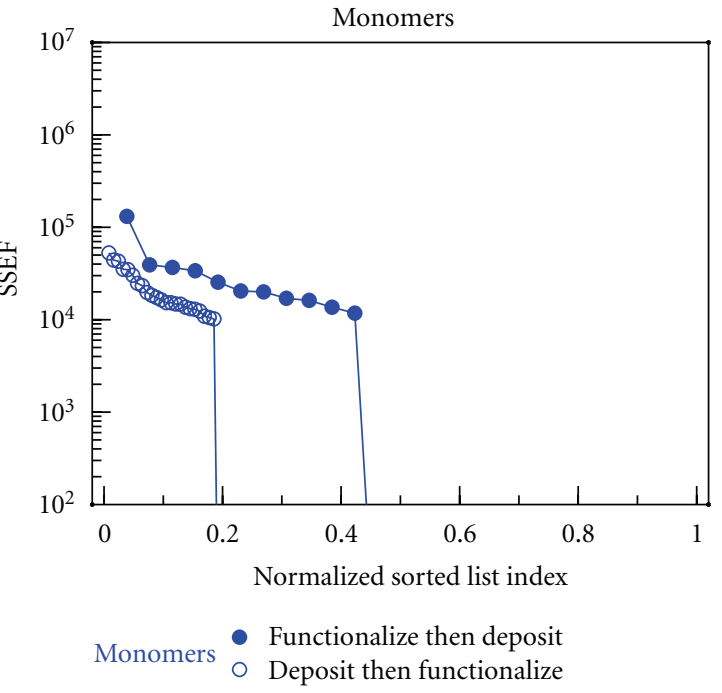

(b)

FIGURE 8: Pre- and post-deposition functionalization of silver nanocubes. Sorted SSEF data from (a) silver nanocube dimers and (b) silver nanocube monomers. The categories are FF dimer with no gaps (black), non-FF dimers with no gaps (red), and monomers (blue). Excitation wavelength: $633 \mathrm{~nm}$. Nanocube size: $110 \mathrm{~nm}$.

the Raman dye before they were allowed to cluster. Briefly, the silver nanocubes were dispersed in a $1 \mathrm{mM}$ ethanoic solution of 4-aminothiophenol. After 3 hours, the nanocubes were separated and cleaned by repeated centrifugation in ethanol and in DI water. These functionalized nanocubes were deposited on substrates coated with a patterned PMMA layer (with an array of rectangular nanopores) by dropcasting. Following the removal of the PMMA layer, substrates with clusters of nanocubes in predetermined locations were obtained. In these experiments, no electric fields were applied during the deposition of the cubes because we had found that the Raman reporter molecule is not compatible with the electrophoretic deposition conditions. As a result, a comparatively low number of clusters were obtained for this study, most of them monomers and dimers.

Figure 8 presents the comparison between SERS data obtained with pre-deposition functionalization and postdeposition functionalization of the silver nanocubes with the Raman reporter. The SERS data from dimers were grouped according to cluster configuration (FF versus non-FF). The trendlines for FF dimers indicate that the collective SSEFs are higher when the functionalization precedes the deposition. Still, the same is true for non-FF dimers and even more significantly for monomers. The trendline for FF dimers is still lower than that of non-FF dimers, even after pre-deposition functionalization. Taken together, these observations suggest that there is an increase in the surface concentration of the Raman dye in pre- versus post-deposition functionalized nanocubes. However, the similarities in the results recorded for monomers and for FF dimers suggest that the most significant difference in the surface concentration is in the gaps between the nanocubes and the silicon substrate. These gaps are narrower than the lateral gaps in the FF dimers, are presumably the least accessible to the solution, and are the most plausible location for the hot-spots in monomers. The increase in the SSEF values of all the clusters regardless of cluster configuration (Figure 8) can be accounted for by the increase in the surface concentration of 4-aminothiophenol in all the gaps between silver nanocubes and the silicon substrate. The fact that the increase in SSEF values is the least significant in the non-FF dimers could indicate that the molecules in the junctions between the nanocubes are significantly more radiant than the molecules on the bottom surface of these dimers. The hypothesis that lower SSEF values in FF dimers are due to lower surface concentration of molecules in the gap is probably incorrect. Other hypotheses, relating the SSEF values to the effect of cluster configuration on the plasmon resonance frequencies and the field intensity distribution around the nanocubes, are more plausible and should be examined further [23].

\section{Conclusions}

The analysis of the distribution of the SSEF values is a direct method to probe the reliability of an SERS substrate design with respect to fabrication tolerances. We have demonstrated the acquisition of SERS signals from hundreds of SERS substrates, each consisting of a small cluster of nanoparticles and produced by self-assembly techniques (either vertical deposition or electrophoretic deposition using patterned substrates). The analysis of the distribution of the SSEF values from these substrates provided insightful information regarding the important parameters that lead to high and reproducible SSEFs using silver nanocubes. 
Our results reproduce the SSEF dependence on the polarization of the incident laser beam commonly reported in the literature. The azimuth angle studies indicate that linear clusters of silver nanocubes should be prepared with their long axis parallel to the laser polarization. This linear alignment was achieved by clustering the nanocubes within rectangular nanopores.

By increasing the cluster size, from monomers to tetramers, the average SSEF values increased, both with $633 \mathrm{~nm}$ and $785 \mathrm{~nm}$ incident laser light. Furthermore, the distribution of SSEF values from same-size clusters becomes narrower with increasing cluster size. In contrast, no clear correlation between maximum SSEF value and cluster size could be established from the data.

Our experimentation with various cluster configurations indicates that better sensitivity could be achieved in SERS by avoiding the assembly of nanocubes into close-packed linear clusters with face-to-face arrangement. This guideline for cluster design can be satisfied using our fabrication methods by introducing nanoscale corrugations to the base or the side-walls of the nanopores in the substrate.

For most of the practical applications of SERS in chemical and biomolecule sensing, the plasmonic nanostructured substrate has to be assembled prior to the exposure to the analyte. Adsorption of the analyte following the assembly of the cluster may not lead to the highest possible concentration of molecules on the surface of the nanocubes. The face of the nanocube adjacent to the flat silicon substrate is potentially depleted of Raman reporters, and thus it could be challenging to effectively utilize hot-spots in the gap between the silicon and the silver nanocubes for SERS.

\section{Acknowledgments}

The authors acknowledge financial support from ONR (N000140911190). The support of the Maryland NanoCenter and its NispLab is appreciated. The NispLab is supported in part by the NSF as a MRSEC Shared Experimental Facility.

\section{References}

[1] H. Xu, E. J. Bjerneld, M. Käll, and L. Börjesson, "Spectroscopy of single hemoglobin molecules by surface enhanced raman scattering," Physical Review Letters, vol. 83, no. 21, pp. 43574360, 1999.

[2] J. P. Camden, J. A. Dieringer, Y. Wang et al., "Probing the structure of single-molecule surface-enhanced Raman scattering hot spots," Journal of the American Chemical Society, vol. 130, no. 38, pp. 12616-12617, 2008.

[3] M. Moskovits, "Surface-enhanced Raman spectroscopy: a brief retrospective," Journal of Raman Spectroscopy, vol. 36, no. 6-7, pp. 485-496, 2005.

[4] F. J. García-Vidal and J. B. Pendry, "Collective theory for surface enhanced Raman scattering," Physical Review Letters, vol. 77, no. 6, pp. 1163-1166, 1996.

[5] K. L. Kelly, E. Coronado, L. L. Zhao, and G. C. Schatz, "The optical properties of metal nanoparticles: the influence of size, shape, and dielectric environment," Journal of Physical Chemistry B, vol. 107, no. 3, pp. 668-677, 2003.
[6] I. D. Mayergoyz, D. R. Fredkin, and Z. Zhang, "Electrostatic (plasmon) resonances in nanoparticles," Physical Review B, vol. 72, no. 15, pp. 1-15, 2005.

[7] N. Grillet, D. Manchon, F. Bertorelle et al., "Plasmon coupling in silver nanocube dimers: resonance splitting induced by edge rounding," ACS Nano, vol. 5, no. 12, pp. 9450-9462, 2011.

[8] W. Lee, S. Y. Lee, R. M. Briber et al., "Self-assembled SERS substrates with tunable surface plasmon resonances," Advanced Functional Materials, vol. 21, no. 18, pp. 3424-3429, 2011.

[9] D. K. Lim, K. S. Jeon, H. M. Kim, J. M. Nam, and Y. D. Suh, "Nanogap-engineerable raman-active nanodumbbells for single-molecule detection," Nature Materials, vol. 9, no. 1, pp. 6067, 2010

[10] T. Dadosh, J. Sperling, G. W. Bryant et al., "Plasmonic control of the shape of the raman spectrum of a single molecule in a silver nanoparticle dimer," ACS Nano, vol. 3, no. 7, pp. 19881994, 2009.

[11] Y. Fang, N. H. Seong, and D. D. Dlott, "Measurement of the distribution of site enhancements in surface-enhanced raman scattering," Science, vol. 321, no. 5887, pp. 388-392, 2008.

[12] Y. Sun and Y. Xia, "Shape-controlled synthesis of gold and silver nanoparticles," Science, vol. 298, no. 5601, pp. 21762179, 2002.

[13] I. Ojea-Jimenez, N. G. Bastus, and V. Puntes, "Influence of the sequence of the reagents addition in the citrate-mediated synthesis of gold nanoparticles," Journal of Physical Chemistry C, vol. 115, no. 32, pp. 15752-15757, 2011.

[14] S. Nie and S. R. Emory, "Probing single molecules and single nanoparticles by surface-enhanced Raman scattering," Science, vol. 275, no. 5303, pp. 1102-1106, 1997.

[15] K. Kneipp, Y. Wang, H. Kneipp et al., "Single molecule detection using surface-enhanced Raman scattering (SERS)," Physical Review Letters, vol. 78, no. 9, pp. 1667-1670, 1997.

[16] H. Chen, Z. Sun, W. Ni et al., "Plasmon coupling in clusters composed of two-dimensionally ordered gold nanocubes," Small, vol. 5, no. 18, pp. 2111-2119, 2009.

[17] J. A. Fan, C. Wu, K. Bao et al., "Self-assembled plasmonic nanoparticle clusters," Science, vol. 328, no. 5982, pp. 1135$1138,2010$.

[18] L. Chuntonov and G. Haran, "Trimeric plasmonic molecules: the role of symmetry," Nano Letters, vol. 11, no. 6, pp. 2440 2445, 2011.

[19] F. S. Ou, M. Hu, I. Naumov et al., "Hot-spot engineering in polygonal nanofinger assemblies for surface enhanced Raman spectroscopy," Nano Letters, vol. 11, no. 6, pp. 2538-2542, 2011.

[20] S. C. Yang, H. Kobori, C. L. He et al., "Plasmon hybridization in individual gold nanocrystal dimers: direct observation of bright and dark modes," Nano Letters, vol. 10, no. 2, pp. 632637, 2010.

[21] D. S. Kim, J. Heo, S. H. Ahn, S. W. Han, W. S. Yun, and Z. H. Kim, "Real-space mapping of the strongly coupled plasmons of nanoparticle dimers," Nano Letters, vol. 9, no. 10, pp. 36193625, 2009.

[22] K. L. Wustholz, A. I. Henry, J. M. McMahon et al., "Structureactivity relationships in gold nanoparticle dimers and trimers for surface-enhanced raman spectroscopy," Journal of the American Chemical Society, vol. 132, no. 31, pp. 10903-10910, 2010.

[23] S. Y. Lee, L. Hung, G. S. Lang, J. E. Cornett, I. D. Mayergoyz, and O. Rabin, "Dispersion in the SERS enhancement with silver nanocube dimers," ACS Nano, vol. 4, no. 10, pp. 57635772, 2010. 
[24] G. Chen, Y. Wang, M. Yang et al., "Measuring ensemble-averaged surface-enhanced Raman scattering in the hotspots of colloidal nanoparticle dimers and trimers," Journal of the American Chemical Society, vol. 132, no. 11, pp. 3644-3645, 2010.

[25] M. Rycenga, P. H. C. Camargo, W. Li, C. H. Moran, and Y. Xia, "Understanding the SERS effects of single silver nanoparticles and their dimers, one at a time," Journal of Physical Chemistry Letters, vol. 1, no. 4, pp. 696-703, 2010.

[26] J. M. Romo-Herrera, R. A. Alvarez-Puebla, and L. M. LizMarzán, "Controlled assembly of plasmonic colloidal nanoparticle clusters," Nanoscale, vol. 3, no. 4, pp. 1304-1315, 2011.

[27] J. A. Liddle, Y. Cui, and P. Alivisatos, "Lithographically directed self-assembly of nanostructures," Journal of Vacuum Science and Technology B, vol. 22, no. 6, pp. 3409-3414, 2004.

[28] K. D. Alexander, M. J. Hampton, S. Zhang, A. Dhawan, H. Xu, and R. Lopez, "A high-throughput method for controlled hotspot fabrication in SERS-active gold nanoparticle dimer arrays," Journal of Raman Spectroscopy, vol. 40, no. 12, pp. 2171-2175, 2009.

[29] Y. Yin, Y. Lu, B. Gates, and Y. Xia, “Template-assisted selfassembly: a practical route to complex aggregates of monodispersed colloids with well-defined sizes, shapes, and structures," Journal of the American Chemical Society, vol. 123, no. 36, pp. 8718-8729, 2001.

[30] Q. Zhang, T. Xu, D. Butterfield et al., "Controlled placement of CdSe nanoparticles in diblock copolymer templates by electrophoretic deposition," Nano Letters, vol. 5, no. 2, pp. 357$361,2005$.

[31] S. Y. Lee and O. Rabin, "A unique solid-solid transformation of silver nanoparticles on reactive-ion-etching processed silicon," Nanotechnology, vol. 23, no. 6, article 065301, 2012.

[32] L. Besra and M. Liu, "A review on fundamentals and applications of electrophoretic deposition (EPD)," Progress in Materials Science, vol. 52, no. 1, pp. 1-61, 2007.

[33] N. Chandrasekharan and P. V. Kamat, "Assembling gold nanoparticles as nanostructured films using an electrophoretic approach," Nano Letters, vol. 1, no. 2, pp. 67-70, 2001.

[34] M. A. Islam, Y. Xia, M. L. Steigerwald et al., "Addition, suppression, and inhibition in the electrophoretic deposition of nanocrystal mixture films for CdSe nanocrystals with $\gamma-\mathrm{Fe}_{2} \mathrm{O}_{3}$ and Au Nanocrystals," Nano Letters, vol. 3, no. 11, pp. 16031606, 2003.

[35] R. C. Bailey, K. J. Stevenson, and J. T. Hupp, "Assembly of micropatterned colloidal gold thin films via microtransfer molding and electrophoretic deposition," Advanced Materials, vol. 12, no. 24, pp. 1930-1934, 2000.

[36] E. C. Le Ru, E. Blackie, M. Meyer, and P. G. Etchegoint, "Surface enhanced raman scattering enhancement factors: a comprehensive study," Journal of Physical Chemistry C, vol. 111, no. 37, pp. 13794-13803, 2007.

[37] L. Rodríguez-Lorenzo, R. A. Álvarez-Puebla, I. PastorizaSantos et al., "Zeptomol detection through controlled ultrasensitivesitive surface-enhanced raman scattering," Journal of the American Chemical Society, vol. 131, no. 13, pp. 4616-4618, 2009.

[38] A. Tao, P. Sinsermsuksakul, and P. Yang, "Polyhedral silver nanocrystals with distinct scattering signatures," Angewandte Chemie, vol. 45, no. 28, pp. 4597-4601, 2006.

[39] M. Inoue and K. Ohtaka, "Surface enhanced Raman scattering by metal spheres. I. Cluster effect," Journal of the Physical Society of Japan, vol. 52, no. 11, pp. 3853-3864, 1983.
[40] D. A. Genov, A. K. Sarychev, V. M. Shalaev, and A. Wei, "Resonant field enhancements from metal nanoparticle arrays," Nano Letters, vol. 4, no. 1, pp. 153-158, 2004.

[41] P. H. C. Camargo, L. Au, M. Rycenga, W. Li, and Y. Xia, "Measuring the SERS enhancement factors of dimers with different structures constructed from silver nanocubes," Chemical Physics Letters, vol. 484, no. 4-6, pp. 304-308, 2010. 

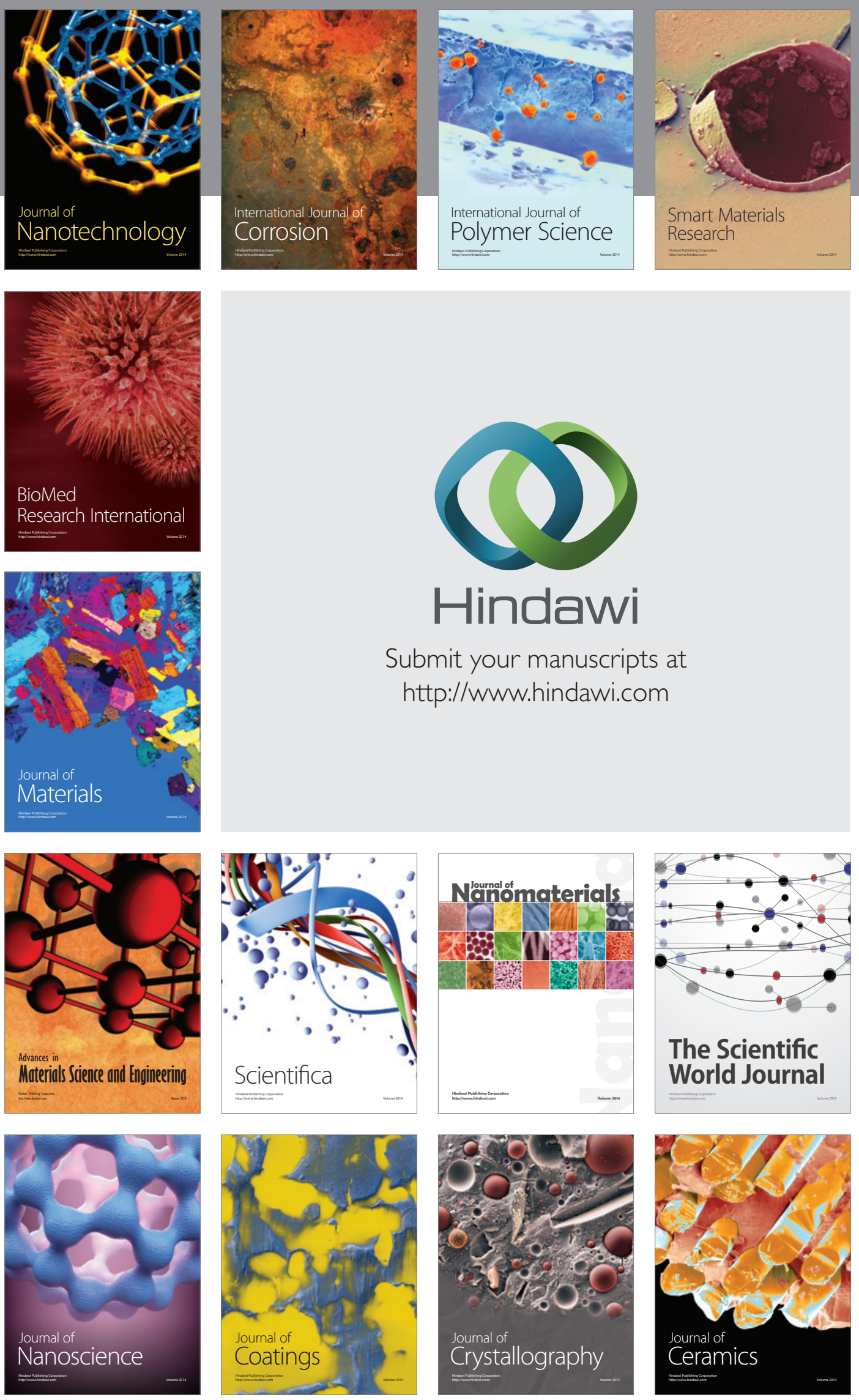

The Scientific World Journal

Submit your manuscripts at

http://www.hindawi.com

\section{World Journal}

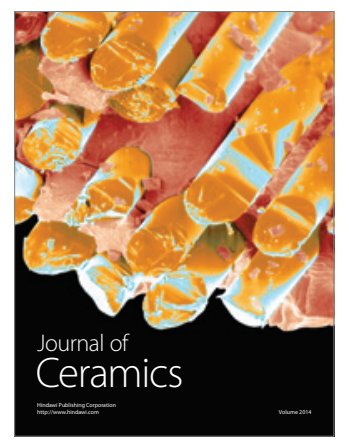

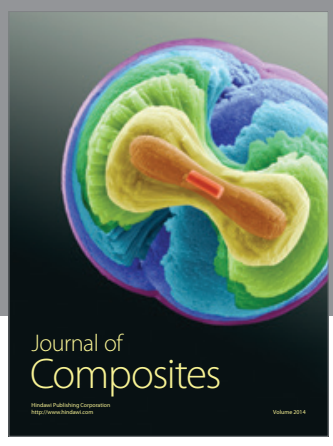
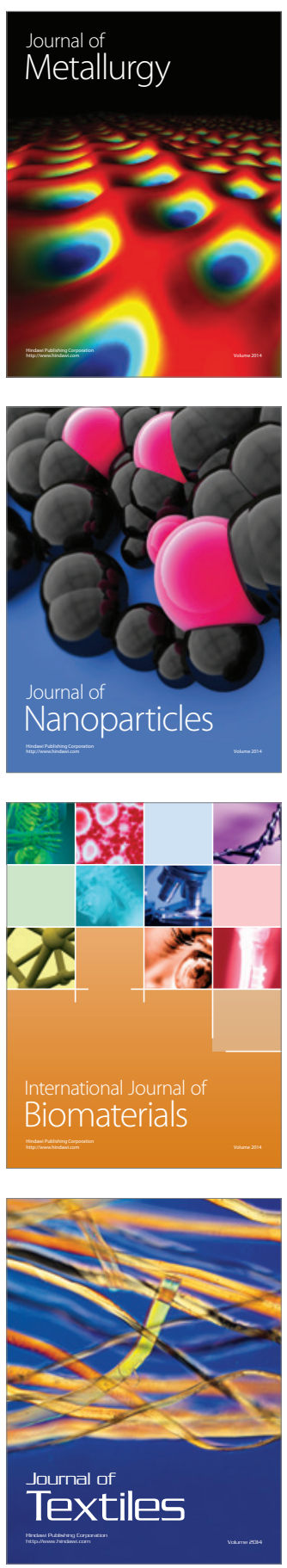